\title{
Effect of Early Nephrology Referral on the Initiation of Hemodialysis and Survival in Patients With Chronic Kidney Disease and Cardiovascular Diseases
}

\author{
Satoko Nakamura, MD; Hiroto Nakata, MD; Fumiki Yoshihara, MD; Kei Kamide, MD; \\ Takeshi Horio, MD; Hajime Nakahama, MD; Yuhei Kawano, MD
}

\begin{abstract}
Background The timing of referral to nephrologists is highly variable in patients with chronic kidney disease (CKD). The impact of early referral on the timing of hemodialysis (HD) and mortality in the patients with CKD and cardiovascular diseases (CVD) was evaluated in this present study.

Methods and Results A total of 366 patients with CKD and CVD began HD at the National Cardiovascular Center between 1983 and 2003, and survival was followed until 2005. The times between the first evaluation by a nephrologist and the date of the first HD were categorized as late (LR $<6$ months) or early (ER $\geq 6$ months) referral. Patients were classified into the ER $(n=194)$ and LR $(n=172)$ groups. Clinical data and renal function were not different. In the LR group, the renal function declined more rapidly and the duration between the first visit to the hospital and the first HD was shorter than the ER group. The survival rate after the initiation of HD was better in the ER group. Age, pre end-stage renal disease therapy and cardiac function had a significant impact on survival. Conclusions Early nephrology referral is important and necessary for for patients with CKD and CVD in terms of a better renal prognosis and survival. (Circ J 2007; 71: 511-516)
\end{abstract}

Key Words: Cardiovascular disease; Chronic kidney disease; Dialysis; Nephrology referral; Survival

$\mathbf{T}$ he number of patients with end-stage renal disease (ESRD) in Japan has increased almost linearly since an individually pursued survey began in 1983, reaching 197,213 at the end of 1999! The gross annual mortality percentage was almost $9 \%$ in Japan, and was lower than that of Western countries. Indeed, in the USA and Europe, mortality within the first 3 months of starting renal replacement therapy (RRT) was disproportionately high, between 6 and $16 \%, 2,3$ and represented $32 \%$ of deaths occurring within the first year of RRT in 1 series and $47 \%$ of deaths in the first 2 years of RRT in another.

Cardiovascular disease (CVD) has been the main cause of morbidity and mortality in patients undergoing RRT, accounting for approximately $50 \%$ of deaths 1,3 because patients newly introduced to dialysis have been older, and the primary diseases leading to hemodialysis were diabetic nephropathy. The risk of cardiac events, such as myocardial infarction, in patients undergoing RRT has been estimated by 2 of the largest ESRD registries, the United States Renal Data System and the European registry of patients on RRT, to be between 3.5- and 50-fold higher than in the general population. ${ }^{4}$ Risk factors for CVD in the general population have been well characterized5 The Framingham Study and other studies have identified a number of atherogenic risk factors, including increasing age, male gender, family his-

(Received October 6, 2006; revised manuscript received January 9, 2007; accepted January 23, 2007)

Division of Hypertension and Nephrology, Department of Medicine, National Cardiovascular Center, Suita, Japan

Mailing address: Satoko Nakamura, MD, Division of Hypertension and Nephrology, National Cardiovascular Center, 5-7-1 Fujishiro-dai, Suita 565-8565, Japan. E-mail: snakamur@hsp.ncvc.go.jp tory, hypertension, diabetes mellitus, smoking and elevated serum cholesterol levels. More recently, homocysteine and lipoprotein(a) were identified as additional coronary risk factors, necessitating a broadening of existing risk factor reduction strategies. Many of these traditional risk factors are also found in patients with renal failure6 We also reported the role of blood pressure, as one of the most important risk factors for CVD, in determining the prognosis for hemodialysis patients? The chronic kidney disease (CKD) was reported as a risk factor for cardiovascular death in the general population, and the adequate usage of antihypertensive medication such as angiotensin II receptor blocker had cardio-renal protection in patients undergoing coronary angiography?

However, little attention has been focused on patients with chronic renal insufficiency in the pre-ESRD period, a time when the risk-factor burden is increasing and specific risk factor reduction strategies could be implemented. Although several published studies have evaluated mortality predictors in maintenance dialysis patients, few have explored the impact on mortality risk of medical care provided to patients before the start of ESRD. It is tempting to speculate that patients referred to a renal clinic early in the pre-ESRD period might experience a greater survival benefit compared with those who are referred later. Early placement of vascular access, thereby avoiding the need for catheter placement and its associated complications, as well as the earlier control of hypertension, renal anemia, acidosis and hyperphosphatemia, are potential mechanisms through which this benefit might be achieved. Recent studies suggested that early predialysis nephrological care was associated with a lower mortality in hemodialysis 
Table 1 Clinical Characteristics of the Patients

\begin{tabular}{|c|c|c|c|}
\hline Patient characteristics & $\begin{array}{c}\text { All patients } \\
(n=366)\end{array}$ & $\begin{array}{c}L R \\
(n=172)\end{array}$ & $\begin{array}{c}E R \\
(n=194)\end{array}$ \\
\hline Age at first hemodialysis (years old) & $63 \pm 13$ & $63 \pm 13$ & $64 \pm 12$ \\
\hline $\operatorname{Sex}(\%$ men $)$ & 78 & 80 & 76 \\
\hline \multicolumn{4}{|l|}{ Cause of ESRD (\%) } \\
\hline Diabetes mellitus & 33 & $40^{*}$ & 27 \\
\hline Chronic glomerulonephritis & 33 & $26^{*}$ & 39 \\
\hline Nephrosclerosis & 23 & 22 & 24 \\
\hline Others & 11 & 12 & 9 \\
\hline \multicolumn{4}{|l|}{ Comobid conditions ( $\%$ yes) } \\
\hline Hypertension & 98 & 96 & 100 \\
\hline Diabetes & 55 & 52 & 58 \\
\hline Hyperlipidemia & 39 & 39 & 40 \\
\hline$I H D$ & 60 & 58 & 63 \\
\hline CVA & 30 & 33 & 28 \\
\hline$P V D$ & 11 & 14 & 9 \\
\hline$A A$ & 15 & 19 & 12 \\
\hline Smoking & 64 & 63 & 65 \\
\hline \multicolumn{4}{|l|}{ Laboratory parameters at initiation of hemodialysis } \\
\hline Serum albumin $(g / d l)$ & $3.4 \pm 0.5$ & $3.4 \pm 0.5$ & $3.5 \pm 0.5$ \\
\hline Hemoglobin $(\mathrm{g} / \mathrm{dl})$ & $8.2 \pm 1.5$ & $8.1 \pm 1.6$ & $8.3 \pm 1.5$ \\
\hline Serum phosphorus ( $\mathrm{mg} / \mathrm{dl})$ & $6.3 \pm 2.1$ & $6.2 \pm 2.3$ & $6.4 \pm 1.9$ \\
\hline Serum calcium $(\mathrm{mg} / \mathrm{dl})$ & $8.2 \pm 1.0$ & $8.1 \pm 0.9$ & $8.2 \pm 1.1$ \\
\hline Serum creatinine $(\mathrm{mg} / \mathrm{dl})$ & $10.0 \pm 3.4$ & $9.6 \pm 3.6$ & $10.6 \pm 3.9$ \\
\hline Blood urea nitrogen $(\mathrm{mg} / \mathrm{dl})$ & $96 \pm 30$ & $93 \pm 28$ & $99 \pm 31$ \\
\hline Total cholesterol $(\mathrm{mg} / \mathrm{dl})$ & $174 \pm 44$ & $174 \pm 49$ & $174 \pm 40$ \\
\hline HDL-cholesterol $(\mathrm{mg} / \mathrm{dl})$ & $39 \pm 14$ & $36 \pm 13 * *$ & $41 \pm 14$ \\
\hline SBP at initiation of hemodialysis $(\mathrm{mmHg})$ & $160 \pm 27$ & $161 \pm 30$ & $160 \pm 25$ \\
\hline$D B P$ at initiation of hemodialysis $(\mathrm{mmHg})$ & $79 \pm 15$ & $79 \pm 17$ & $79 \pm 14$ \\
\hline FS on echocardiography (\%) & $33 \pm 11$ & $31 \pm 11 *$ & $34 \pm 11$ \\
\hline LVMI on echocardiography $\left(\mathrm{g} / \mathrm{m}^{2}\right)$ & $192 \pm 61$ & $202 \pm 64 *$ & $184 \pm 58$ \\
\hline Asynergy of left ventricle wall on echocardiography (\% yes) & 33 & $41 * *$ & 25 \\
\hline \multicolumn{4}{|l|}{ Conditions at first hemodialysis (\% yes) } \\
\hline With heart failure & 49 & 47 & 51 \\
\hline With infection & 10 & $14^{*}$ & 7 \\
\hline After the operations & 8 & $14^{\# \#}$ & 2 \\
\hline After the catheter procedures & 5 & $8^{*}$ & 3 \\
\hline
\end{tabular}

Data refer to the mean $\pm S D$.

${ }^{*} p<0.05, * * p<0.01,{ }^{*} p<0.005$, \# $p<0.001$ vs $E R$.

$L R$, late nephrologist referral; ER, early nephrologist referral; ESRD, end-stage renal disease; IHD, ischemic heart disease; CVA, cerebrovascular disease; $P V D$, peripheral vascular disease; AA, aortic aneurysm; HDL, high-density lipoprotein; SBP, systolic blood pressure; DBP, diastolic blood pressure; FS, fractioning shortening; LVMI, left ventricular mass index.

Table 2 Clinical Characteristics of the Patients

\begin{tabular}{|c|c|c|c|}
\hline Parameters & All patients & $L R$ & $E R$ \\
\hline Age at first visit to hospital (years old) & $57 \pm 12$ & $58 \pm 13$ & $56 \pm 12$ \\
\hline Time between first visit to the hospital and first hemodialysis (months) & $91.7 \pm 78.4$ & $71.0 \pm 72.0^{\#}$ & $104.0 \pm 79.6$ \\
\hline Time of first evaluation by a nephrologist and first hemodialysis (months) & $27.9 \pm 50.3$ & $1.2 \pm 21.7^{\# \#}$ & $51.5 \pm 59.9$ \\
\hline Serum creatinine at first visit to the hospital ( $m g / d l)$ & $2.2 \pm 1.8$ & $2.4 \pm 2.4$ & $2.0 \pm 1.3$ \\
\hline Serum creatinine at first evaluation by a nephrologist $(\mathrm{mg} / \mathrm{dl})$ & $3.6 \pm 2.4$ & $5.7 \pm 3.0^{\# \#}$ & $2.7 \pm 1.5$ \\
\hline Antihypertensive medications before first hemodialysis (\% yes) & 91 & $84^{\# \#}$ & 96 \\
\hline$C C B(\%$ yes $)$ & 82 & $71^{\# \#}$ & 90 \\
\hline ACEI (\% yes) & 22 & $16^{*}$ & 26 \\
\hline$\beta$-blockers (\% yes) & 41 & $30^{\#}$ & 50 \\
\hline Diuretics (\% yes) & 71 & 67 & 73 \\
\hline Pre-ESRD medication for acidosis (\% yes) & 40 & $18^{\# \#}$ & 57 \\
\hline Pre-ESRD medication for uremia (\% yes) & 14 & $5^{\# \#}$ & 20 \\
\hline Pre-ESRD medication for hyperuricemia (\% yes) & 44 & $23^{\#}$ & 60 \\
\hline Pre-ESRD medication for hyperphosphatemia (\% yes) & 36 & $16^{\# \#}$ & 50 \\
\hline Pre-ESRD use of Vitamin D (\% yes) & 7 & $3 *$ & 18 \\
\hline Pre-ESRD medication for hyperkalemia (\% yes) & 16 & $9^{\#}$ & 21 \\
\hline Pre-ESRD use of erythropoietin (\% yes) & 25 & 9 9\# & 34 \\
\hline Blood transfusion (\% yes) & 4 & $7^{*}$ & 2 \\
\hline Placement of permanent vascular access before first hemodialysis (\% yes) & 57 & $31^{\# \#}$ & 73 \\
\hline Use of temporary catheter at first hemodialysis (\% yes) & 45 & $72^{\# \#}$ & 30 \\
\hline Time between placement of vascular access and first hemodialysis (days) & $48 \pm 106$ & $8 \pm 34^{\# \#}$ & $65 \pm 121$ \\
\hline
\end{tabular}

Data refer to the mean $\pm S D$.

${ }^{*} p<0.05, * * p<0.01,{ }^{\#} p<0.005,{ }^{\#} p<0.001$ vs $E R$.

$C C B$, calcium channel blockers; ACEI, angiotensin converting enzyme inhibitor. Other abbreviations as in Table 1. 
patients in the USA ${ }^{10-12}$ The purpose of this study was to explore the impact of early nephrology referral (ER) before ESRD initiation on the timing of hemodialysis therapy and patient survival in Japanese patients.

\section{Methods}

\section{Study Population}

Three hundred and sixty-six patients (286 males and 80 females) with chronic renal failure, who had been introduced to hemodialysis therapy at the National Cardiovascular Center Hospital between 1983 and 2003 and discharged to branch hospitals for maintenance hemodialysis, were studied retrospectively. The time between first evaluation by a nephrologist and the date of first hemodialysis is referred as 'the time of evaluation'. The time of evaluation was less than 1 year in $59 \%$ of patients, less than 6 months in $47 \%$ and less than 3 months in $37 \%$. Next, the patients were classified into 2 groups according to the time of evaluation and were categorized as late (less than 6 months) or early (more than 6 months).

The characteristics of the patient cohort at the time of the initiation of hemodialysis and during the pre-ESRD period are shown in Tables 1 and 2. The primary renal diseases that caused chronic renal failure included 120 cases of chronic glomerulonephritis, 122 cases of diabetic nephropathy, and 85 cases of nephrosclerosis, 12 cases of polycystic kidney disease, 2 cases of lupus nephritis and 25 cases of unknown origin.

The ischemic heart disease (IHD) included myocardial infarction and angina pectoris; myocardial infarction was diagnosed using either electrocardiograms or echocardiograms, and angina pectoris was diagnosed from both symptoms and electrocardiograms. Cerebral vascular disease (cerebral infarction and hemorrhage) (CVA) was diagnosed by the clinical history, neurological examination and computerized tomography findings. Peripheral vascular disease (PVD) was diagnosed by symptoms (exertional claudication, rest pain) and/or prior revascularization procedures to the legs, and/or an ankle/brachial pressure index less than 0.9. A thoracic or abdominal aortic aneurysm (AA) was diagnosed by the clinical history and the computed tomography or ultrasonography findings. Smoking habits were recorded in 2 categories: current/former smokers and non-smokers.

Mortality findings were collected up to 30 June 2005 from the time when the patients were admitted for initiation of hemodialysis. Answers from 365 respondents were collected and the recovery rate was $99.7 \%$. Of these patients, 167 died during the observation period (41 months). The primary end-point was all causes of mortality. Causes of death were obtained from the death certificates and hospital record forms. The deceased patients had chronic glomerulonephritis in 34 cases, diabetes in 64 cases, nephrosclerosis in 45 cases, polycystic kidney disease in 3 cases, lupus nephritis in 2 cases and unknown diseases in 19 cases.

\section{Risk Factors Examined}

The risk factors examined included: age, primary renal disease (diabetic nephropathy or not), past history of CVD, blood pressure, laboratory parameters, pre-ESRD therapy and placement of vascular access. Nurses measured blood pressure at the initiation phase of hemodialysis with the patient in the supine position after resting for more than $5 \mathrm{~min}$ before starting the first dialysis session. Antihypertensive medications were used in $91 \%$ of the patients.

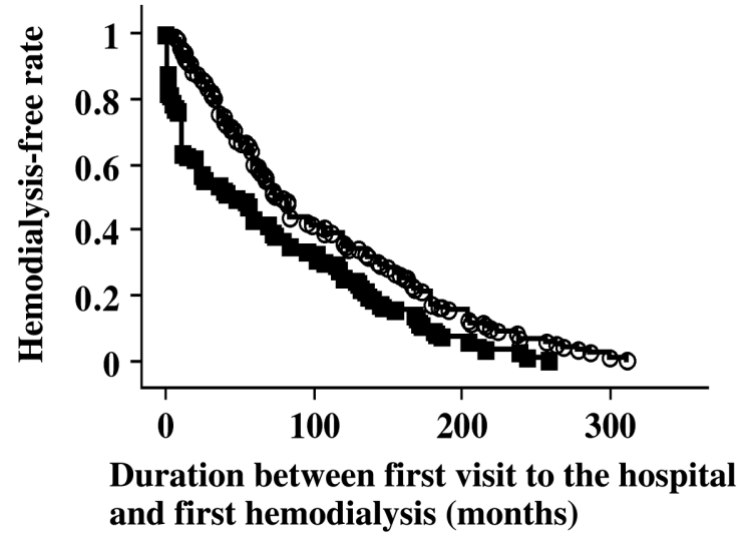

Fig 1. Cumulative survival curves for the development of end-stage renal disease in hemodialysis patients; early referral group (open circles) and late referral group (closed squares) $(\mathrm{p}<0.005)$.

\section{Statistical Analysis}

Values are represented as mean \pm SD with a $\mathrm{p}$ value of less than 0.05 taken to indicate statistical significance. To compare continuous and categorical variables, $t$-tests and chi-square tests were used as appropriate. The association between risk factors and the timing of hemodialysis was determined by logistic regression analysis. Cumulative survival curves were estimated by using the Kaplan-Meier analysis and compared using the Cox-Montel (log-rank) test. Univariate survival analysis was carried out using the Kaplan-Meier product-limit method. Prognostic factors of survival were identified using the Cox's proportional hazards regression model. Age, primary renal disease, CVD, serum albumin concentrations, blood pressure and early nephrologist referral were included as variables of the multivariate models. All statistical analyses were performed with Stat View version 5.0 (SAS Institute Inc).

\section{Results}

\section{Patient Characteristics}

Tables 1 and 2 show the baseline characteristics of all patients. The mean age at first hemodialysis was 63 years old and $60 \%$ of patients had IHD. Age at the first hemodialysis and gender were not different between the ER group and the late nephrologist referral (LR) group. ESRD caused by chronic glomerulonephritis was evident in the ER group, while diabetes mellitus was evident in the LR group $(\mathrm{p}<$ 0.05). The number of patients with IHD, CVA, PVD or AA was not different between the 2 groups. All of them had at least 1 CVD, such as hypertension, IHD, CVA, PVD or AA. Blood pressure at the initiation phase, blood urea nitrogen, creatinine, albumin, total cholesterol, trigricerides, glucose, serum calcium and phosphate were not significantly different between the groups. High-density lipoprotein-cholesterol was higher in the ER group. The echocardiography findings were worse in the LR group.

Table 2 shows that age and serum creatinine at the first visit to the hospital were not different between the 2 groups, and that the time between the first visit to the hospital and the first hemodialysis was significantly longer in the ER group $(\mathrm{p}<0.005)$. The decline in renal function was slower in the ER group. The use of antihypertensive medications was more evident in the ER group, except for diuretics. PreESRD medications for acidosis (sodium bicarbonate), ure- 
Table 3 Univariate Risk Factors for the Initiation of Hemodialysis Therapy Based on Logistic Regression Analysis

\begin{tabular}{lccc}
\hline \hline Risk factors & $R R$ & $95 \%$ CI & p value \\
\hline Age at first visit to the hospital (years old) & 1.04 & $1.02-1.05$ & $<0.0001$ \\
Serum creatinine at first visit to the hospital (mg/dl) & 1.71 & $1.56-1.88$ & $<0.0001$ \\
ER (yes) & 0.65 & $0.49-0.85$ & $<0.005$ \\
No visit to the hospital (yes) & 2.61 & $1.28-5.32$ & $<0.01$ \\
Antihypertensive medications (no) & 2.01 & $1.06-3.85$ & $<0.05$ \\
ACEI (no) & 1.48 & $1.09-2.02$ & $<0.05$ \\
B-blockers (no) & 1.37 & $1.05-1.79$ & $<0.05$ \\
Pre-ESRD medication for acidosis (no) & 1.32 & $0.99-1.75$ & 0.06 \\
Pre-ESRD medication for uremia (no) & 1.80 & $1.11-2.94$ & $<0.05$ \\
Pre-ESRD medication for hyperuricemia (no) & 1.44 & $1.09-1.92$ & $<0.05$ \\
Pre-ESRD medication for hyperphosphatemia (no) & 1.43 & $1.08-1.88$ & $<0.05$ \\
Pre-ESRD use of Vitamin D (no) & 1.45 & $0.74-2.83$ & 0.28 \\
Pre-ESRD mediation for hyperkalemia (no) & 1.98 & $0.91-2.96$ & 0.13 \\
Pre-ESRD use of erythropoietin (no) & 1.97 & $1.41-2.76$ & $<0.0001$ \\
Past history of cardiovascular diseases (yes) & 0.99 & $0.75-1.32$ & 0.98 \\
Diabetes mellitus (yes) & 0.99 & $0.76-1.29$ & 0.95 \\
Placement of permanent vascular access before first hemodialysis (yes) & 0.84 & $0.63-1.12$ & 0.23 \\
\hline
\end{tabular}

$R R$, relative risk; $C I$, confidence interval. Other abbreviations as in Tables 1,2.

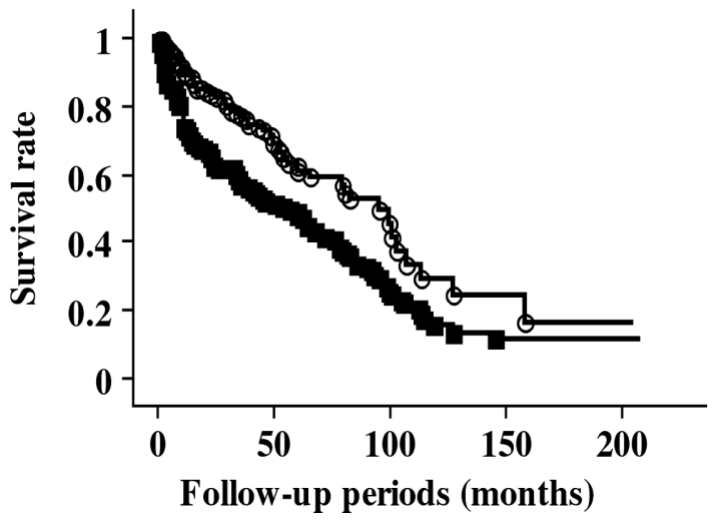

Fig 2. Cumulative survival curves for all causes-mortality of hemodialysis patients; early referral group (open circles) and late referral group (closed squares) $(\mathrm{p}<0.0001)$.

mia (spherical carbonaceous adsorbent), hyperphosphatemia (precipitate calcium carbonate), hyperuricemia (allopurinol), hyperkalemia (calcium polystyrene sulfonate, sodium polystyrene sulfonate) and anemia (erythropoietin) were more evident in the ER group, and blood transfusion was evident in the LR group. The permanent vascular access was placed more frequently in the ER group (73\%) and earlier in the ER group ( 65 days before the first hemodialysis).

\section{Development of ESRD}

As shown in Fig 1, cumulative survival curves for the development of ESRD were computed. The duration was between the date of the first visit to the hospital and the date of first hemodialysis. There was a significant difference between the ER group and the LR group $(p<0.005)$. Patients in the LR group were introduced to hemodialysis therapy earlier than those in the ER group. Table 3 shows the effect of several factors on the prolongation of initiation of hemodialysis therapy using univariate analysis. Renal function at first visit at the hospital, early referral to a nephrologist, antihypertensive medications and pre-ESRD medications had an impact on the prolongation of initiation of hemodialysis, but the past history of CVD, diabetic mellitus and permanent vascular access did not.
Morbidity, Mortality and Relative Risks (RRs)

Of the 366 consecutive hemodialysis patients, 167 died during the follow-up period of $41 \pm 42$ months. As shown in Fig 2, the cumulative survival curves were computed. There was a significant difference between the ER group and the LR group $(\mathrm{p}<0.0001)$. Univariate analysis for all causes of mortality was performed (Table 4$)$. The age at initiation of hemodialysis $(p<0.0001)$, history of CVD ( $p<$ $0.001)$, blood pressure $(\mathrm{p}<0.05)$, early referral to a nephrologist $(\mathrm{p}<0.01)$ and cardiac function $(\mathrm{p}<0.05)$ were identified as being significantly associated with survival. Diabetes mellitus was not a significant risk. Univariate analysis for all causes of mortality at 1 year was performed (Table 4). Age at initiation of hemodialysis $(p<0.0005)$, history of CVD $(\mathrm{p}<0.01)$, blood pressure $(\mathrm{p}<0.005)$, early referral to a nephrologist $(\mathrm{p}<0.01)$ and early placement of permanent vascular access $(\mathrm{p}<0.05)$ were identified as being significantly associated with survival at 1 year.

According to the Cox's proportional hazards regression analysis, multivariate analysis for all causes of mortality was performed using the following risk factors: age, ER, diabetes mellitus, past history of CVD, systolic blood pressure and serum albumin (Table 5). The significant variables retained by the models were age $(\mathrm{p}<0.0001)$ and ER $(\mathrm{p}<$ 0.05 ). Multivariate analysis for all causes of mortality was performed using the risks of: age, pre-ESRD medications, erythropoietin, antihypertensive medications, and on whether the patient had diabetes mellitus, a past history of CVD, and what their systolic blood pressure and serum albumin levels were (data not shown). The significant variables retained by the models were age (RR $1.05, \mathrm{p}<0.0001)$, past history of CVD (RR 1.79, $\mathrm{p}<0.05)$, inadequate using of erythropoietin (RR 1.94, p<0.05), and inadequate using of antihypertensive medications (RR 2.35, p<0.005).

\section{Discussion}

We retrospectively examined the effect of nephrologist referral on the initiation of hemodialysis therapy and the mortality rate in CKD patients with CVD. ER had an impact on the prolongation of hemodialysis therapy initiation and a better survival rate to compare the LR, even if age and comorbidity were not different. In the present study, we found that LR to a nephrologist is: (1) a common problem 
Table 4 Univariate Risk Factors for 1 Year and All Cause Mortality of Hemodialysis Patients Based on Cox's Proportional Hazards Regression Model

\begin{tabular}{|c|c|c|c|c|}
\hline \multirow{2}{*}{ Risk factors } & \multicolumn{2}{|c|}{1 year mortality } & \multicolumn{2}{|c|}{ All cause mortality } \\
\hline & $R R(95 \% C I)$ & p value & $R R(95 \% C I)$ & $p$ value \\
\hline Age at first hemodialysis (years old) & $1.05(1.02-1.08)$ & $<0.001$ & $1.05(1.04-1.07)$ & $<0.0001$ \\
\hline ER (yes) & $0.43(0.23-0.80)$ & $<0.01$ & $0.54(0.46-0.88)$ & $<0.01$ \\
\hline Antihypertensive medications (no) & $0.98(0.30-3.20)$ & 0.98 & $1.68(1.01-2.81)$ & $<0.05$ \\
\hline Pre-ESRD medication for acidosis (no) & $1.32(0.65-2.72)$ & 0.45 & $1.34(0.91-1.98)$ & 0.14 \\
\hline Pre-ESRD medication for uremia (no) & $1.27(0.60-2.51)$ & 0.27 & $2.77(1.13-6.80)$ & $<0.05$ \\
\hline Pre-ESRD medication for hyperuricemia (no) & $0.69(0.35-1.35)$ & 0.28 & $0.98(0.68-1.43)$ & 0.93 \\
\hline Pre-ESRD medication for hyperphosphatemia (no) & $1.90(0.86-4.19)$ & 0.11 & $1.56(1.08-2.06)$ & $<0.05$ \\
\hline Pre-ESRD use of erythropoietin (no) & $2.73(0.84-8.94)$ & 0.10 & $1.80(1.01-3.27)$ & $<0.05$ \\
\hline Past history of CVD (yes) & $2.97(1.32-6.70)$ & $<0.01$ & $1.94(1.32-2.86)$ & $<0.001$ \\
\hline Diabetes mellitus (yes) & $0.76(0.40-1.44)$ & 0.40 & $1.32(0.96-1.82)$ & 0.09 \\
\hline Placement of permanent vascular access (yes) & $0.34(0.14-0.79)$ & $<0.05$ & $0.76(0.46-1.24)$ & 0.26 \\
\hline SBP at initiation $(\mathrm{mmHg})$ & $0.98(0.97-0.99)$ & $<0.005$ & $0.99(0.98-0.99)$ & $<0.05$ \\
\hline DBP at initiation $(\mathrm{mmHg})$ & $0.96(0.94-0.99)$ & $<0.001$ & $0.98(0.97-0.99)$ & $<0.0001$ \\
\hline Serum creatinine at initiation phase of hemodialysis $(\mathrm{mg} / \mathrm{dl})$ & $0.84(0.75-0.93)$ & $<0.005$ & $0.89(0.84-0.93)$ & $<0.0001$ \\
\hline Serum albumin $(\mathrm{mg} / \mathrm{dl})$ & $0.42(0.21-0.81)$ & $<0.01$ & $0.76(0.53-1.09)$ & 0.13 \\
\hline Hemoglobin $(\mathrm{mg} / \mathrm{dl})$ & $1.33(1.10-1.61)$ & $<0.005$ & $1.12(1.02-1.25)$ & $<0.05$ \\
\hline Hematocrit (\%) & $1.09(1.02-1.16)$ & $<0.01$ & $1.04(1.01-1.08)$ & $<0.05$ \\
\hline HDL-cholesterol $(\mathrm{mg} / \mathrm{dl})$ & $1.00(0.98-1.03)$ & 0.77 & $0.98(0.97-1.00)$ & $<0.05$ \\
\hline First hemodialysis with heart failure (yes) & $1.56(0.82-2.97)$ & 0.17 & $1.60(1.05-2.44)$ & $<0.05$ \\
\hline First hemodialysis with infection (yes) & $3.34(1.52-7.32)$ & $<0.01$ & $2.73(1.33-5.63)$ & $<0.01$ \\
\hline First hemodialysis after the operations (yes) & $3.93(1.65-9.39)$ & $<0.005$ & $3.77(1.55-9.16)$ & $<0.005$ \\
\hline First hemodialysis after the catheter procedures (yes) & $1.27(0.36-4.55)$ & 0.71 & $1.08(0.43-2.72)$ & 0.87 \\
\hline FS $(\%)$ & $0.97(0.94-1.00)$ & 0.12 & $0.97(0.94-0.99)$ & $<0.05$ \\
\hline $\operatorname{LVMI}\left(\mathrm{g} / \mathrm{m}^{2}\right)$ & $1.00(0.99-1.01)$ & 0.89 & $1.01(1.00-1.01)$ & $<0.05$ \\
\hline Asynergy of left ventricle wall on the cardiac echocardiograms (yes) & $7.27(3.18-16.62)$ & $<0.0001$ & $2.60(1.52-4.48)$ & $<0.0005$ \\
\hline
\end{tabular}

CVD, cardiovascular diseases. Other abbreviations as in Tables 1,3.

in our hospital; (2) associated with an earlier start to hemodialysis therapy; (3) associated with a greater prevalence of poor outcome predictors, including comorbidity and cardiac function; (4) associated with suboptimal care in the preESRD period, especially for those using antihypertensive medications, pre-ESRD medications, erythropoietin and with a lack of permanent vascular access at the start of hemodialysis therapy; and (5) contributes significantly to increased mortality independent of other known mortality predictors.

In the USA, delayed referral of patients to a nephrologist is common; more than $52.2 \%$ of patients have their first encounter with a nephrologist within 1 year of dialysis therapy initiation $!^{2}$ Of much greater concern, a substantial proportion of patients $(32 \%)$ had their first appointment with a nephrologist less than 4 months before the start of ESRD and were consequently provided with little time to manage the complications of advanced uremia and prepare for RRT. Our findings showed that $59.6 \%$ of patients had their first contact with a nephrologist within 1 year of dialysis therapy initiation, and corroborated the results of other USA and European investigators who found similarly high rates of LR in recently published studies ${ }^{13,14}$ These observations suggest that primary care providers and non-renal specialists refer patients with renal insufficiency late in the course of their disease to nephrologists, thereby delaying the provision of pre-ESRD health care. These practices might be caused, in part, by a lack of consensus by nephrologists on the optimal time for the referral of patients with chronic renal insufficiency. Recently, we showed that renal parenchymal damage was evident in patients with $\mathrm{CVD}^{15}$ and the deterioration in renal function after aortic surgery was dependent on the preoperative renal function, proteinuria and renal size ${ }^{16}$ For the population who had CVD, creatinine clearance less than $60 \mathrm{ml} / \mathrm{min}$, proteinuria (containing microalbuminuria) or small kidneys, ER might be necessary.
Table 5 Multivariate Risk Factors for Survival of Hemodialysis Patients Based on Cox's Proportional Hazards Regression Model

\begin{tabular}{lccc}
\hline \hline Risk factors & RR & $95 \%$ CI & p value \\
\hline Age at first hemodialysis (years old) & 1.05 & $1.03-1.07$ & $<0.0001$ \\
ER (yes) & 0.69 & $0.48-0.98$ & $<0.05$ \\
Diabetes mellitus $($ yes $)$ & 1.19 & $0.84-1.67$ & 0.32 \\
Past history of CVD $($ yes $)$ & 1.38 & $0.92-2.08$ & 0.13 \\
SBP at initiation $(\mathrm{mmHg})$ & 1.00 & $0.99-1.01$ & 0.67 \\
Serum albumin at initiation $(\mathrm{mg} / \mathrm{dl})$ & 0.85 & $0.58-1.23$ & 0.39 \\
\hline
\end{tabular}

Abbreviations as in Tables 1,3,4.

We hypothesized that the delayed referral of patients to a nephrologist in the pre-ESRD period would compromise patient care delivery during this period. In support of this, we found that the LR patients were less likely to be prescribed antihypertensive medications, pre-ESRD medications or erythropoietin during the pre-ESRD period. Anemia, a strong predictor of mortality, was not prevalent among the LR patients, but the transfusion of red blood cells was performed more often in LR patients. We also highlight the strikingly lower degree of placement of permanent vascular access and higher degree of dialysis catherters in the LR group.

One could easily speculate that the poor delivery of nephrological care during this critical pre-ESRD period might have adverse effects for the patient outcomes. When patients are referred for late pre-ESRD care, there is a failure to use measures to halt the progression of renal insufficiency. The optimal blood pressure control, optimal control of anemia and several metabolic derangements, and creation of permanent vascular access are considered as factors for better survival10,12 In our studies, the LR patients started hemodialysis therapy earlier than the ER patients, even if the renal function at the time of the first visit to the hospital 
and the comorbidity were not different between them. This means that the renal function declined rapidly in the LR patients compared with the ER patients. There was no difference in the decline in renal function between diabetic and non-diabetic patients. Age and renal dysfunction, inadequate usage or under use of pre-ESRD medications were risk factors for the earlier initiation of hemodialysis therapy.

The detrimental consequence of LR on patient mortality is the principal finding from this study. LR patients had significantly poorer survival compared with ER patients. Using Cox's proportional hazard regression analysis, LR remained independently predictive. In previous studies, LR patients were more common among black men than white men, uninsured patients than insured patients, and patients with severe comorbid diseases than those with mild comorbid diseases 10,12 In Japan, there are few ethnic differences and there is universal health insurance. In the present study, there was no difference in comorbidity between the groups.

In the study, the association of LR with an elevated mortality risk was seen in patients with ESRD both with and without diabetes. The impact of LR on mortality was the same in the non-diabetic and diabetic cohorts. The previous study showed that no difference in survival was observed between the LR and ER patients with diabetes, and suggested that the diabetic patients were more likely to be followed up by internists and 1 or more subspecialists during the pre-ESRD period and therefore receive more intensive health care compared with the non-diabetic patients in the USA $!^{2}$ In Japan, there is universal health-care insurance and patients were followed up by internists or subspecialists during the pre-ESRD period; therefore, diabetic patients were not likely to be followed up more frequently compared with non-diabetic patients.

We found that ER was a significant risk reduction factor for survival, while diabetes and a past history of CVD were not. As for the pre-ESRD medications, using erythropoietin and antihypertensive medications were independent factors for better survival. Recent studies showed that erythropoietin reduces renal dysfunction ${ }^{17}$ and can improve cardiac function, renal function and quality of life in patients with chronic heart failure and chronic renal failure ${ }^{18}$ Our use of erythropoietin in the pre-ESRD period was not different compared with the results of recent studies in the USA, but nevertheless seems to be low. Pre-ESRD therapy involving the use of eythropoietin or antihypertensive medications seems to be important for the prognosis of patients with renal failure. A nephrologist is expected to have good communication with other medical doctors for the management of patients with renal failure, especially in terms of anemia and blood pressure.

We conclude that LR to a nephrologist is a common occurrence for new dialysis patients in our hospital, and is associated with suboptimal pre-ESRD care. LR patients experienced a greater degree of blood transfusion and a lower prevalence of permanent vascular access at dialysis therapy initiation, substantial under-use of antihypertensive medications, substantial under-use of pre-ESRD medications, and substantial under-use of erythropoietin during the pre-ESRD period. Moreover, even among ER patients, the overall prevalence of permanent vascular access placement and pre-ESRD erythropoietin use was low. LR patients experienced an earlier initiation of hemodialysis therapy and significantly poorer survival than ER patients. The findings of the present study suggest the need for improved communication between nephrologists and other healthcare providers, given the importance of ER for renal clinic patients, so that greater measures can be taken to arrest the progression of renal disease and promote a healthier transition to dialysis therapy. They also suggest that greater attention be given to anemia management and the use of arterio venous fistulas during the pre-ESRD period to improve patient survival.

\section{References}

1. Nakai S, Shinzato T, Nagura Y, Masakane I, Kitaoka T, Shinoda T, et al. An overview of regular dialysis treatment in Japan (as of Dec 31, 2001). Ther Apher Dial 2004; 8: 3-32.

2. Khan IH, Catto GRD, Edward N, Fleming LW, Henderson IS, MacLeod AM. Influence of coexisting disease on survival on renalreplacement therapy. Lancet 1993; 341: 415-418.

3. US Renal Data System. Patient mortality and survival in ESRD. Am J Kidney Dis 1999; 34: S74-S86.

4. Foley RN, Parfrey PS, Sarnak MJ. Clinical epidemiology of cardiovascular disease in chronic renal disease. Am J Kidney Dis 1998; 32: S12-S119.

5. Kannel WB, Dawber TR, Kagan A. Factors of risk in the development of coronary artery disease: Six-year follow-up experience: The Framingham study. Ann Intern Med 1960; 55: 33-50.

6. Parfrey PS, Foley RN, Harnett JD, Kent GM, Murray D, Barre PE. Outcome and risk factors of ischemic heart disease in chronic uremia. Kidney Int 1996; 49: 1428-1434.

7. Kimura G, Tomita J, Nakamura S, Uzu T, Inenaga T. Interaction between hypertension and other cardiovascular risk factors in survival of hemodialyzed patients. Am J Hypertens 1996; 9: 1006-1012.

8. Nakamura K, Okamura T, Hayakawa T, Kadowaki T, Kita Y, Ohnishi $\mathrm{H}$, et al; The NIPPON DATA90 Research Group. Chronic kidney disease is a risk factor for cardiovascular death in a community-based population in Japan: NIPPON DATA90. Circ J 2006; 70: 954-959.

9. Ikeda N, Nishimura S, Kyo S, Komiyama N, Matsumoto K, Inoue T, et al. Valsaltan cardio-renal protection in patients undergoing coronary angiography complicated with chronic renal insufficiency (VAL-CAPR) trial: Rationale and design. Circ J 2006; 70: 548-552.

10. Avon J, Bohn RL, Levy E, Levin R, Owen WF Jr, Winkelmayer WC, et al. Nephrologist care and mortality in patients with chronic renal insufficiency. Arch Intern Med 2002; 162: 2002-2006.

11. Kinchen KS, Sadler J, Brookmeyer R, Klag MJ, Levey AS, Powe $\mathrm{NR}$, et al. The timing of specialist evaluation in chronic kidney disease and mortality. Ann Intern Med 2002; 137: 479-486.

12. Stack AG. Impact of timing of nephrology referral and pre-ESRD care on mortality risk among new ESRD patients in the United States. Am J Kid Dis 2003; 41: 310-318.

13. Astor BC, Eustace JA, Powe NR, Klag MJ, Sadler JH, Fink NE, et al. Timing of nephrologist referral and arteriovenous access use: The CHOICE Study. Am J Kid Dis 2001; 38: 494-501.

14. Fink JC, Blahunt SA, Reddy M, Light PD. Use of erythropoietin before the initiation of dialysis and its impact on mortality. Am J Kid Dis 2001; 37: 348-355.

15. Fujii H, Nakamura S, Kuroda S, Yoshihara F, Nakahama H, Inenaga $\mathrm{T}$, et al. Relationship between renal artery stenosis and intrarenal damage in autopsy subjects with stroke. Nephrol Dial Transplant 2006; 21: 113-119.

16. Nakamura S, Yoshihara F, Kamide K, Nakahama H, Nishigami K, Fukuchi K, et al. Microalbuminuria and deterioration of renal function after elective repair of infrarenal abdominal aortic aneurysm. Clin Nephrol 2006; 65: 165-172.

17. Chatterjee PK. Pleiotropic renal actions of erythropoietin. Lancet 2005; 365: 1890-1892.

18. Iaina A, Silverberg DS, Wexler D. Therapy insight: Congestive heart failure, chronic kidney disease and anemia, the cardio-renal-anemia syndrome. Nat Clin Pract Cardiovasc Med 2005; 2: 95-100. 